\title{
Nucleotide excision repair pathway gene polymorphisms are linked to breast cancer risk in a Chinese population
}

\author{
Bang-shun $\mathrm{He}^{1}$, Tao Xü , Yu-qin Pan ${ }^{1}$, Han-jin Wang ${ }^{2}$, William C. Cho ${ }^{3}$, Kang Lin ${ }^{1}$, \\ Hui-ling Sun ${ }^{1}$, Tian-yi Gao ${ }^{4}$, Shu-kui Wang ${ }^{1}$ \\ ${ }^{1}$ General Clinical Research Center, Nanjing First Hospital, Nanjing Medical University, Nanjing, China \\ ${ }^{2}$ Department of General Surgery, Nanjing First Hospital, Nanjing Medical University, Nanjing, China \\ ${ }^{3}$ Department of Clinical Oncology, Queen Elizabeth Hospital, Hong Kong, China \\ ${ }^{4}$ Department of Laboratory Medicine, Nanjing First Hospital, Nanjing Medical University, Nanjing, China \\ Correspondence to: Shu-kui Wang, email: shukwang@163.com \\ Keywords: association study, breast cancer, Chinese females, nucleotide excision repair (NER) pathway, polymorphism \\ Received: May 26, $2016 \quad$ Accepted: October 10, $2016 \quad$ Published: October 19, 2016
}

\section{ABSTRACT}

Polymorphisms in nucleotide excision repair (NER) pathway genes are associated with the risk of breast cancer, but the relevance of these associations appeared to vary according to the ethnicity of the subjects. To systemically evaluate the potential associations between NER polymorphisms and breast cancer risk in a Chinese population, we carried out a case-control study on 450 breast cancer patients and 430 healthy controls. Sequenom MassARRAY was used for genotyping, and immunohistochemistry was performed to detect estrogen receptor (ER), progesterone receptor (PR), and human epidermal growth factor receptor 2 (HER-2) expression in tumor tissue. Our results showed that ERCC1 rs11615 (additive model: OR adjusted: 1.36, 95\% CI: 1.08-1.71, $p=0.009$ ), XPC rs2228000 (additive model: OR adjusted $_{1.39,}$, $15 \%$ CI: 1.13-1.72, $p=0.002$ ) and ERCC2/XPD rs50872 (additive model: $\mathrm{OR}_{\text {adjusted }}: 1.32$, $95 \%$ CI: 1.04-1.67, $p=0.021$ ) were associated with an increased risk of breast cancer. Stratified analysis revealed three polymorphisms (rs11615, rs1800975, and rs50872) to be associated with breast cancer in menopausal females. Three polymorphisms were associated with specific breast cancer grades (rs11615 with grade 3, rs2228000 and rs50872 with grade 1-2). Two polymorphisms (rs2228001 and rs50872) were associated with the risk of breast cancer with negative lymph node involvement. rs1800975 and rs50872 were associated with the risk of ER- and PR- breast cancer, whereas rs11615 was associated with the risk of $\mathrm{ER}^{+}$and $\mathrm{PR}^{+}$breast cancer. We found that carriers of the T allele of ERCC1 rs11615, XPC rs2228000 and rs50872, particularly in postmenopausal females, have an increased risk of breast cancer.

\section{INTRODUCTION}

Breast cancer is a complex multifactorial disease with unclear etiology. DNA damage and genomic instability, a potential risk of breast cancer, are induced by common environmental factors [2]. However, we are born with a system to protect our genome from DNA damage and correct for damage after it occurs, including nucleotide excision repair (NER), mismatch repair (MMR), bases excision repair (BER), transcription-coupled repair (TCR), and double-strand DNA break repair systems [3].
NER repairs damage introduced by ultraviolet (UV) radiation, products of organic combustion, intrastrand DNA cross-links, heavy metals, and oxidative stress. Several proteins, including ERCC1, XPA, XPB/ ERCC3，XPC，XPD/ERCC2，ERCC4/XPF， ERCC5/ $\mathrm{XPG}$, and $\mathrm{PE} / \mathrm{DDB} 1$, are involved in the repair process, maintaining genome integrity to prevent carcinogenesis. The process of NER comprises several distinct steps, including DNA damage recognition, DNA damage demarcation, damaged DNA incision, repair patch synthesis, and ligation. Polymorphisms in NER pathway 
Table 1: Candidate genes and polymorphisms

\begin{tabular}{|c|c|c|c|c|c|}
\hline Gene & rs \# & Chromosome & $\begin{array}{c}\text { Allele } \\
\text { (major/minor) }\end{array}$ & Position & HWE in controls \\
\hline$X P A$ & rs1800975 & 9:97697296 & $\mathrm{G} / \mathrm{A}$ & $5^{\prime}$ non-coding region $(-4 \mathrm{~A} / \mathrm{G})$ & $0.118 / 2.445$ \\
\hline$E R C C 1$ & rs 11615 & $19: 45420395$ & $\mathrm{C} / \mathrm{T}$ & Exon 4 (Asn118Asn) & $0.509 / 0.436$ \\
\hline$X P C$ & rs 2228000 & $3: 14158387$ & $\mathrm{C} / \mathrm{T}$ & Exon 9 (Ala499Val) & $0.498 / 0.460$ \\
\hline$X P C$ & rs2228001 & $3: 14145949$ & $\mathrm{~A} / \mathrm{C}$ & Exon 16 (Gln939Lys) & $0.267 / 1.231$ \\
\hline$E R C C 2 / X P D$ & rs238406 & $19: 45365051$ & $\mathrm{G} / \mathrm{T}$ & Exon 6 (Arg156Arg) & $0.766 / 0.088$ \\
\hline$E R C C 2 / X P D$ & rs1799793 & $19: 45364001$ & $\mathrm{G} / \mathrm{A}$ & Exon 10 (Asp 312 Asn) & $0.101 / 2.687$ \\
\hline$E R C C 2 / X P D$ & rs50872 & $19: 45359191$ & $\mathrm{C} / \mathrm{T}$ & Intron 12 & $0.945 / 0.005$ \\
\hline$E R C C 2 / X P D$ & rs13181 & $19: 45351661$ & $\mathrm{~T} / \mathrm{A}$ & Exon 23 ( Lys751 Gln) & $0.716 / 0.132$ \\
\hline$E R C C 2 / X P D$ & rs3810366 & $19: 45370684$ & $\mathrm{C} / \mathrm{G}$ & Promoter (-114) & $0.099 / 2.728$ \\
\hline$E R C C 4 / X P F$ & rs1799801 & $16: 13948101$ & $\mathrm{~T} / \mathrm{C}$ & Exon 11 (Ser835Ser) & $0.619 / 0.247$ \\
\hline$E R C C 5 / X P G$ & rs 17655 & $13: 102875652$ & $\mathrm{C} / \mathrm{G}$ & Exon 15 (His 1104Asp) & $0.077 / 3.137$ \\
\hline
\end{tabular}

genes have been associated with increased risk for a number of cancers [4] [5-8].

Breast cancer patients and their relatives tend to have constitutively low NER levels in their peripheral blood lymphocytes [9, 10]. Moreover, polymorphisms in NER pathway genes have been linked to breast cancer risk in studies conducted on patients of some ethnicities. However, the conclusions have been inconsistent [11-14]. Among the Chinese population, studies have reported correlations between polymorphisms in NER pathway genes and breast cancer risk, but to date, there is no systematic investigation on the genetic susceptibility of the NER pathway in breast cancer [15-19]. To provide a more comprehensive understanding of the relationships between specific polymorphisms in the NER pathway genes (Table 1) on the carcinogenesis of breast cancer, we performed a breast cancer risk association study and a meta-analysis.

\section{RESULTS}

None of the tested polymorphisms deviated from Hardy-Weinberg equilibrium (HWE) in controls (Table 1). There were no significant differences in the age and menopausal status among cases and controls (Table 2).

The genotype distribution in the two groups and their subgroups of menopausal status are presented in Table 3. The result showed that ERCC1 rs11615, XPC rs2228000, and ERCC2/XPD rs50872 carriers have a higher breast cancer risk in the whole study population. Stratified analysis of menopausal status revealed that $X P C$ rs2228000 has a higher breast cancer risk in the premenopausal sub-cohort. While in the postmenopausal sub-cohort, ERCC1 rs11615 and ERCC2/XPD rs50872 were associated with increased breast cancer risk. On the contrary, XPA rs1800975 and XPC rs2228001 were associated with decreased breast cancer risk.

Based on the observed significant associations, we then performed stratified analysis based on pathological characteristics of the breast cancer (tumor size, lymph node involvement) and expression of specific proteins in tumor tissue (PR, ER, and HER-2). Tumor size (T3T4) was associated with all polymorphisms of interest except for XPA rs1800975. In addition, ERCC1 rs11615 carriers have a high risk of breast cancer with grade 3 , while $X P C$ rs2228000 and ERCC2/XPD rs50872 are linked to a high risk for breast cancer with grades 1 and 2, respectively. For the lymph node involvement subgroup, $X P C$ rs2228001 and ERCC2/XPD rs50872 carriers have a high risk of breast cancer with negative lymph node involvement. While ERCC1 rs11615 and XPC rs2228000, were significantly associated with both negative and positive lymph node involvement subgroups (Table 4).

For tumor tissue characteristics, XPA rs 1800975 and $E R C C 2 / X P D$ rs50872 carriers have a high risk of breast cancer with negative expression of ER and PR. While ERCC1 rs11615 have a high risk of ER+ and $\mathrm{PR}+$ breast cancer and PR. The susceptibility of XPC rs2228000 to breast cancer risk was observed in both subgroups; however, there was no significant association for $X P C$ rs2228001 in any subgroup (Table 5).

For ERCC2/XPD rs238406, rs1799793, rs13181, rs3810366, ERCC4/XPF rs1799801, ERCC5/XPG rs17655, no significant association was found (Table 3).

To confirm the results of our case study, we performed a meta-analysis involving XPC rs2228000, rs2228001, XPA rs1800975, and ERCC1 rs11615 (Table 6). We identified 14 studies for the meta-analysis according to 
Table 2: Clinical characteristics of the participants

\begin{tabular}{|c|c|c|c|}
\hline & Cases, $n(\%)$ & Controls, $n(\%)$ & $P$ value \\
\hline Age (mean \pm SD) & $52.85 \pm 10.77$ & $52.67 \pm 10.78$ & $0.799 *$ \\
\hline Menopausal status & & & 0.110 \\
\hline Pre- & $206(45.78)$ & $220(51.16)$ & \\
\hline Post- & $244(54.22)$ & $210(48.84)$ & \\
\hline \multicolumn{4}{|l|}{ Tumor size (T1-T4) } \\
\hline $\mathrm{T} 1-\mathrm{T} 2$ & $312(69.33)$ & & \\
\hline $\mathrm{T} 3-\mathrm{T} 4$ & $138(30.67)$ & & \\
\hline \multicolumn{4}{|c|}{ Tumor grade (G1-G3) } \\
\hline G1 & $86(19.11)$ & & \\
\hline G2 & $238(52.89)$ & & \\
\hline G3 & $126(28.00)$ & & \\
\hline \multicolumn{4}{|c|}{ Lymph node involvement } \\
\hline Yes & $235(52.22)$ & & \\
\hline No & $215(47.78)$ & & \\
\hline \multicolumn{4}{|l|}{$\mathbf{E R}$} \\
\hline Positive & $278(61.78)$ & & \\
\hline Negative & $172(38.22)$ & & \\
\hline \multicolumn{4}{|l|}{ PR } \\
\hline Positive & $238(52.89)$ & & \\
\hline Negative & $212(47.11)$ & & \\
\hline \multicolumn{4}{|l|}{ HER-2 } \\
\hline Positive & $353(78.44)$ & & \\
\hline Negative & $97(21.55)$ & & \\
\hline
\end{tabular}

*Independent $t$ test applied to age; ER, estrogen receptor; HER-2, human epidermal growth factor receptor-2; PR, progesterone receptor.

the inclusion criteria. The characteristics of the selected studies are presented in Supplemental Table S1. The allele frequencies of the four polymorphisms in Asian and Caucasian populations are shown in Supplemental Table S2, indicating the allele frequencies of this study were consistent with those of the pooled data.

Pooled results suggested that XPC rs2228000 TT was associated with increased breast cancer risk. In addition, in the Asian population subgroup, XPC rs2228000 TT genotype was a risk factor for breast cancer (Table 6). Similarly, in the population-based studies subgroup, XPC rs2228000 TT genotype was correlated with an increased risk of breast cancer (Table 6). For XPC rs2228001, no significant association was found by pooled or subgroup analysis.

For XPA rs1800975, there were no significant associations with breast cancer risk in the pooled results or the Asian population subgroup; however, in the other ethnic population subgroup, a weak but significant association with increased breast cancer was observed in both the co-dominant and dominant models (Table 6). For ERCC1 rs11615, the pooled results indicated that TT and TT/CT genotype were associated with increased breast cancer risk (Table 6).

\section{DISCUSSION}

This case-control association study revealed that ERCC1 rs11615 (T allele), XPC rs2228000 (T allele) and $E R C C 2 / X P D$ rs50872 (T allele) were associated with increased breast cancer risk. Besides, ERCC1 rs11615 (T allele), and $E R C C 2 / X P D$ rs50872 (T allele) were associated with postmenopausal breast cancer, while $X P C$ 
Table 3: Distribution of the genotypes in the participants and sub-groups

\begin{tabular}{|c|c|c|c|c|c|c|c|c|c|}
\hline \multirow{2}{*}{ Genotype } & \multicolumn{3}{|c|}{ All participants } & \multicolumn{3}{|c|}{ Premenopause } & \multicolumn{3}{|c|}{ Postmenopause } \\
\hline & $\mathrm{Ca} / \mathrm{Co}$ & OR $(95 \% \text { CI })^{*}$ & $P$ value & $\mathrm{Ca} / \mathrm{Co}$ & OR $(95 \% \text { CI })^{\#}$ & $P$ value & $\mathrm{Ca} / \mathrm{Co}$ & OR $(95 \% \text { CI })^{\#}$ & $P$ value \\
\hline \multicolumn{10}{|l|}{$X P A$ rs 1800975} \\
\hline GG & $115 / 93$ & Reference & & $47 / 55$ & Reference & & $68 / 38$ & Reference & \\
\hline GA & $235 / 231$ & $0.82(0.59,1.14)$ & 0.245 & $106 / 114$ & $1.06(0.66,1.71)$ & 0.805 & $129 / 117$ & $0.63(0.39,1.01)$ & 0.057 \\
\hline $\mathrm{AA}$ & $100 / 106$ & $0.77(0.52,1.13)$ & 0.186 & $53 / 51$ & $1.21(0.70,2.10)$ & 0.489 & $47 / 55$ & $0.48(0.27,0.83)$ & 0.009 \\
\hline GA/AA & $335 / 337$ & $0.81(0.59,1.11)$ & 0.185 & $159 / 165$ & $1.11(0.71,1.74)$ & 0.646 & $176 / 172$ & $0.59(0.37,0.92)$ & 0.020 \\
\hline Additive model & $450 / 430$ & $0.88(0.73,1.07)$ & 0.198 & $206 / 220$ & $1.10(0.84,1.45)$ & 0.488 & $244 / 210$ & $0.70(0.53,0.92)$ & 0.012 \\
\hline \multicolumn{10}{|l|}{ ERCC1 rs11615 } \\
\hline $\mathrm{CC}$ & $230 / 261$ & Reference & & $108 / 128$ & Reference & & $122 / 133$ & Reference & \\
\hline CT & $195 / 151$ & $1.45(1.10,1.92)$ & 0.009 & $86 / 86$ & $1.17(0.79,1.74)$ & 0.430 & $109 / 65$ & $1.80(1.21,2.68)$ & 0.004 \\
\hline TT & $25 / 18$ & $1.56(0.83,2.94)$ & 0.168 & $12 / 6$ & $2.32(0.84,6.41)$ & 0.104 & $13 / 12$ & $1.18(0.52,2.69)$ & 0.700 \\
\hline $\mathrm{CT} / \mathrm{TT}$ & $220 / 169$ & $1.46(1.11,1.91)$ & 0.006 & 98/92 & $1.25(0.85,1.83)$ & 0.260 & $122 / 77$ & $1.69(1.16,2.47)$ & 0.007 \\
\hline Additive model & $450 / 430$ & $1.36(1.08,1.71)$ & 0.009 & $206 / 220$ & $1.29(0.93,1.80)$ & 0.131 & $244 / 210$ & $1.42(1.04,1.95)$ & 0.030 \\
\hline \multicolumn{10}{|l|}{$X P C$ rs 2228000} \\
\hline $\mathrm{CC}$ & $201 / 228$ & Reference & & $86 / 116$ & Reference & & $115 / 112$ & Reference & \\
\hline CT & $198 / 174$ & $1.31(0.99,1.73)$ & 0.061 & $94 / 85$ & $1.51(1.01,2.26)$ & 0.048 & $104 / 89$ & $1.15(0.78,1.69)$ & 0.481 \\
\hline TT & $51 / 28$ & $2.16(1.3,3.57)$ & 0.003 & $26 / 19$ & $1.85(0.96,3.57)$ & 0.065 & $25 / 9$ & $2.69(1.20,6.02)$ & 0.016 \\
\hline $\mathrm{CT} / \mathrm{TT}$ & $249 / 212$ & $1.42(1.09,1.86)$ & 0.010 & $120 / 104$ & $1.57(1.07,2.30)$ & 0.022 & $129 / 98$ & $1.30(0.90,1.88)$ & 0.170 \\
\hline Additive model & $450 / 430$ & $1.39(1.13,1.72)$ & 0.002 & $206 / 220$ & $1.41(1.06,1.88)$ & 0.020 & $244 / 210$ & $1.37(1.02,1.85)$ & 0.038 \\
\hline \multicolumn{10}{|l|}{$X P C$ rs 2228001} \\
\hline AA & $193 / 161$ & Reference & & $86 / 91$ & Reference & & $107 / 70$ & Reference & \\
\hline $\mathrm{AC}$ & $195 / 213$ & $0.76(0.57,1.01)$ & 0.060 & $90 / 100$ & $0.96(0.64,1.45)$ & 0.850 & $105 / 113$ & $0.61(0.41,0.91)$ & 0.015 \\
\hline $\mathrm{CC}$ & $62 / 56$ & $0.91(0.60,1.38)$ & 0.649 & $30 / 29$ & $1.08(0.60,1.96)$ & 0.791 & $32 / 27$ & $0.76(0.42,1.38)$ & 0.364 \\
\hline $\mathrm{AC} / \mathrm{CC}$ & $257 / 269$ & $0.79(0.60,1.04)$ & 0.090 & $120 / 129$ & $0.99(0.67,1.45)$ & 0.944 & $137 / 140$ & $0.64(0.44,0.94)$ & 0.022 \\
\hline Additive model & $450 / 430$ & $0.90(0.74,1.09)$ & 0.275 & $206 / 220$ & $1.02(0.77,1.34)$ & 0.909 & $244 / 210$ & $0.79(0.60,1.04)$ & 0.098 \\
\hline \multicolumn{10}{|c|}{$E R C C 2 / X P D$ rs 238406} \\
\hline GG & $128 / 128$ & Reference & & $55 / 62$ & Reference & & $73 / 66$ & Reference & \\
\hline GT & $227 / 216$ & $1.05(0.77,1.43)$ & 0.763 & $108 / 111$ & $1.09(0.70,1.71)$ & 0.700 & $119 / 105$ & $1.01(0.66,1.55)$ & 0.961 \\
\hline TT & $95 / 86$ & $1.12(0.76,1.64)$ & 0.577 & $43 / 47$ & $1.05(0.60,1.84)$ & 0.855 & $52 / 39$ & $1.19(0.69,2.03)$ & 0.534 \\
\hline GT/TT & $322 / 302$ & $1.07(0.80,1.43)$ & 0.661 & $151 / 158$ & $1.08(0.70,1.65)$ & 0.739 & $171 / 144$ & $1.06(0.71,1.59)$ & 0.772 \\
\hline Additive model & $450 / 430$ & $1.06(0.87,1.28)$ & 0.583 & $206 / 220$ & $1.02(0.78,1.35)$ & 0.866 & $244 / 210$ & $1.08(0.83,1.41)$ & 0.546 \\
\hline \multicolumn{10}{|c|}{ ERCC2/XPD rs 1799793} \\
\hline GG & $380 / 367$ & Reference & & $171 / 192$ & Reference & & $209 / 175$ & Reference & \\
\hline GA & $69 / 63$ & $1.05(0.72,1.52)$ & 0.800 & $35 / 28$ & $1.41(0.82,2.42)$ & 0.211 & $34 / 35$ & $0.80(0.48,1.34)$ & 0.399 \\
\hline AA & $1 / 0$ & -- & -- & $0 / 0$ & -- & -- & $1 / 0$ & -- & -- \\
\hline GA/AA & $70 / 63$ & $1.06(0.73,1.54)$ & 0.743 & $35 / 28$ & $1.41(0.82,2.42)$ & 0.211 & $35 / 35$ & $0.82(0.50,1.37)$ & 0.460 \\
\hline Additive model & $450 / 430$ & $1.08(0.75,1.56)$ & 0.683 & $206 / 220$ & $1.41(0.82,2.42)$ & 0.211 & $244 / 210$ & $0.86(0.52,1.41)$ & 0.543 \\
\hline
\end{tabular}

(Continued) 


\begin{tabular}{|c|c|c|c|c|c|c|c|c|c|}
\hline \multirow{2}{*}{ Genotype } & \multicolumn{3}{|c|}{ All participants } & \multicolumn{3}{|c|}{ Premenopause } & \multicolumn{3}{|c|}{ Postmenopause } \\
\hline & $\mathrm{Ca} / \mathrm{Co}$ & OR $(95 \% \mathrm{CI}) *$ & $P$ value & $\mathrm{Ca} / \mathrm{Co}$ & OR $(95 \% \text { CI })^{\#}$ & $P$ value & $\mathrm{Ca} / \mathrm{Co}$ & OR $(95 \% \text { CI })^{\#}$ & $P$ value \\
\hline \multicolumn{10}{|c|}{$E R C C 2 / X P D$ rs 50872} \\
\hline $\mathrm{CC}$ & $269 / 290$ & Reference & & $130 / 151$ & Reference & & $139 / 139$ & Reference & \\
\hline CT & $160 / 126$ & $1.35(1.01,1.79)$ & 0.044 & $66 / 61$ & $1.27(0.83,1.93)$ & 0.270 & $94 / 65$ & $1.42(0.96,2.11)$ & 0.081 \\
\hline TT & $21 / 14$ & $1.64(0.82,3.29)$ & 0.165 & $10 / 8$ & $1.53(0.58,4.01)$ & 0.388 & $11 / 6$ & $1.83(0.66,5.10)$ & 0.245 \\
\hline $\mathrm{CT} / \mathrm{TT}$ & $181 / 140$ & $1.38(1.04,1.81)$ & 0.024 & $76 / 69$ & $1.29(0.86,1.93)$ & 0.212 & $105 / 71$ & $1.46(0.99,2.14)$ & 0.054 \\
\hline Additive model & $450 / 430$ & $1.32(1.04,1.67)$ & 0.021 & $206 / 220$ & $1.25(0.89,1.75)$ & 0.199 & $244 / 210$ & $1.40(1.00,1.95)$ & 0.048 \\
\hline \multicolumn{10}{|c|}{$E R C C 2 / X P D$ rs 13181} \\
\hline TT & $361 / 354$ & Reference & & $169 / 181$ & Reference & & $192 / 173$ & Reference & \\
\hline GT & $86 / 73$ & $1.16(0.82,1.63)$ & 0.412 & $37 / 38$ & $1.06(0.64,1.75)$ & 0.824 & $49 / 35$ & $1.26(0.78,2.03)$ & 0.353 \\
\hline GG & $3 / 3$ & $0.95(0.19,4.74)$ & 0.945 & $0 / 1$ & -- & -- & $3 / 2$ & $1.40(0.23,8.50)$ & 0.715 \\
\hline GT/GG & $89 / 76$ & $1.15(0.82,1.61)$ & 0.432 & $37 / 39$ & $1.03(0.63,1.69)$ & 0.915 & $52 / 37$ & $1.27(0.79,2.02)$ & 0.327 \\
\hline Additive model & $450 / 430$ & $1.13(0.82,1.55)$ & 0.472 & $206 / 220$ & $0.99(0.61,1.62)$ & 0.980 & $244 / 210$ & $1.24(0.81,1.92)$ & 0.326 \\
\hline \multicolumn{10}{|c|}{$E R C C 2 / X P D$ rs 3810366} \\
\hline GG & $112 / 94$ & Reference & & $55 / 56$ & Reference & & $57 / 38$ & Reference & \\
\hline $\mathrm{CG}$ & $234 / 232$ & $0.84(0.60,1.17)$ & 0.292 & $107 / 109$ & $1.00(0.63,1.58)$ & 0.997 & $127 / 123$ & $0.69(0.43,1.12)$ & 0.134 \\
\hline $\mathrm{CC}$ & $104 / 104$ & $0.83(0.56,1.23)$ & 0.353 & $44 / 55$ & $0.80(0.47,1.39)$ & 0.436 & $60 / 49$ & $0.84(0.48,1.48)$ & 0.545 \\
\hline $\mathrm{CG} / \mathrm{CC}$ & $338 / 336$ & $0.84(0.61,1.15)$ & 0.268 & $151 / 164$ & $0.93(0.60,1.44)$ & 0.745 & $187 / 172$ & $0.74(0.47,1.18)$ & 0.202 \\
\hline Additive model & $450 / 430$ & $0.92(0.76,1.11)$ & 0.375 & $206 / 220$ & $0.90(0.69,1.19)$ & 0.462 & $244 / 210$ & $0.93(0.70,1.23)$ & 0.598 \\
\hline \multicolumn{10}{|c|}{$E R C C 4 / X P F$ rs 1799801} \\
\hline TT & $268 / 260$ & Reference & & $118 / 136$ & Reference & & $150 / 124$ & Reference & \\
\hline CT & $157 / 151$ & $1.01(0.76,1.34)$ & 0.949 & $78 / 69$ & $1.31(0.87,1.97)$ & 0.196 & $79 / 82$ & $0.79(0.54,1.17)$ & 0.244 \\
\hline $\mathrm{CC}$ & $25 / 19$ & $1.31(0.70,2.45)$ & 0.399 & $10 / 15$ & $0.76(0.33,1.77)$ & 0.526 & $15 / 4$ & $3.03(0.98,9.37)$ & 0.055 \\
\hline $\mathrm{CT} / \mathrm{CC}$ & $182 / 170$ & $1.04(0.79,1.36)$ & 0.775 & $88 / 84$ & $1.22(0.83,1.79)$ & 0.324 & $94 / 86$ & $0.90(0.62,1.31)$ & 0.579 \\
\hline Additive model & $450 / 430$ & $1.06(0.85,1.33)$ & 0.593 & $206 / 220$ & $1.08(0.79,1.47)$ & 0.647 & $244 / 210$ & $1.05(0.76,1.45)$ & 0.763 \\
\hline \multicolumn{10}{|c|}{$E R C C 5 / X P G$ rs 17655} \\
\hline GG & $101 / 107$ & Reference & & $48 / 61$ & Reference & & $53 / 46$ & Reference & \\
\hline CG & $243 / 233$ & $1.09(0.79,1.52)$ & 0.588 & $114 / 114$ & $1.27(0.80,2.01)$ & 0.311 & $129 / 119$ & $0.94(0.59,1.50)$ & 0.796 \\
\hline $\mathrm{CC}$ & $106 / 90$ & $1.22(0.82,1.80)$ & 0.332 & $44 / 45$ & $1.23(0.70,2.16)$ & 0.471 & $62 / 45$ & $1.20(0.69,2.08)$ & 0.524 \\
\hline $\mathrm{CG} / \mathrm{CC}$ & $349 / 323$ & $1.12(0.82,1.54)$ & 0.464 & $158 / 159$ & $1.26(0.81,1.95)$ & 0.308 & $191 / 164$ & $1.00(0.64,1.57)$ & 1.000 \\
\hline Additive model & $450 / 430$ & $1.11(0.91,1.35)$ & 0.307 & $206 / 220$ & $1.12(0.85,1.48)$ & 0.433 & $244 / 210$ & $1.10(0.83,1.44)$ & 0.509 \\
\hline
\end{tabular}

*Adjusted by age and menopausal status; ${ }^{\#}$ Adjusted by age; Ca, case; Co, control.

rs2228000 (T allele) was associated with premenopausal breast cancer.

The $X P C$ gene encodes a 940 amino acid protein that forms an XPC-RAD23B complex with RAD23B [20]. $X P C$ rs 2228000 is a C-to- $\mathrm{T}$ transition causing a substitution in codon 499 in exon 8 that changes alanine to valine in the interaction domain of XPC with $h H R A D 23$. Consistent with previous reports which linked the TT genotype with lower DNA repair capacity (DRC), [21] this study found that $\mathrm{T}$ allele (CT/TT) carriers have a higher breast cancer risk. An independent study reported that presence of the $X P C$ rs $2228000 \mathrm{~T}$ allele (CT or TT 
Table 4: Polymorphisms on breast cancer risk by pathological characteristics of tumor

\begin{tabular}{|c|c|c|c|c|c|c|c|c|c|c|c|c|c|c|c|c|c|c|c|}
\hline \multirow{2}{*}{ Genotype } & \multirow{2}{*}{ Co } & \multicolumn{3}{|c|}{ Tumor size (T1-T2) } & \multicolumn{3}{|c|}{ Tumor size (T3-T4) } & \multicolumn{3}{|c|}{ Grade (G1-G2) } & \multicolumn{3}{|c|}{ Grade (G3) } & \multicolumn{3}{|c|}{$\begin{array}{l}\text { Lymph node involvement } \\
\text { (Negative) }\end{array}$} & \multicolumn{3}{|c|}{$\begin{array}{l}\text { Lymph node involvement } \\
\text { (Positive) }\end{array}$} \\
\hline & & $\mathrm{Ca}$ & OR $(95 \% \mathrm{CI})^{*}$ & $\begin{array}{c}P \\
\text { value }\end{array}$ & $\mathrm{Ca}$ & OR $(95 \% \mathrm{CI}) *$ & $\begin{array}{c}P \\
\text { value }\end{array}$ & $\mathrm{Ca}$ & OR $(95 \%$ CI $) *$ & $\begin{array}{c}P \\
\text { value }\end{array}$ & $\mathrm{Ca}$ & OR $(95 \%$ CI $) *$ & $\begin{array}{c}P \\
\text { value }\end{array}$ & $\mathrm{Ca}$ & OR $(95 \% \mathrm{CI})^{*}$ & $\begin{array}{c}P \\
\text { value }\end{array}$ & $\mathrm{Ca}$ & OR(95\% CI $)^{*}$ & $\begin{array}{c}P \\
\text { value }\end{array}$ \\
\hline \multicolumn{20}{|c|}{$X P A$ rs 1800975} \\
\hline GG & 93 & 77 & Reference & & 38 & Reference & & 80 & Reference & & 35 & Reference & & 57 & Reference & & 58 & Reference & \\
\hline GA & 231 & 167 & $0.88(0.61,1.26)$ & 0.482 & 68 & $0.71(0.45,1.14)$ & 0.158 & 177 & $0.89(0.62,1.28)$ & 0.535 & 58 & $0.65(0.40,1.06)$ & 0.087 & 111 & $0.78(0.52,1.16)$ & 0.216 & 124 & $0.86(0.58,1.29)$ & 0.470 \\
\hline $\mathrm{AA}$ & 106 & 68 & $0.78(0.51,1.21)$ & 0.267 & 32 & $0.74(0.42,1.27)$ & 0.271 & 67 & $0.74(0.48,1.14)$ & 0.176 & 33 & $0.80(0.46,1.40)$ & 0.432 & 47 & $0.73(0.45,1.17)$ & 0.188 & 53 & $0.80(0.50,1.28)$ & 0.353 \\
\hline $\mathrm{GA} / \mathrm{AA}$ & 337 & 235 & $0.85(0.60,1.20)$ & 0.359 & 100 & $0.73(0.47,1.13)$ & 0.155 & 244 & $0.85(0.60,1.20)$ & 0.350 & 91 & $0.71(0.45,1.12)$ & 0.139 & 158 & $0.77(0.52,1.12)$ & 0.168 & 177 & $0.85(0.58,1.24)$ & 0.395 \\
\hline \multicolumn{20}{|c|}{ ERCCl rs 11615} \\
\hline $\mathrm{CC}$ & 261 & 163 & Reference & & 67 & Reference & & 176 & Reference & & 54 & Reference & & 117 & Reference & & 113 & Reference & \\
\hline $\mathrm{TC}$ & 151 & 128 & $1.36(1.00,1.85)$ & 0.054 & 67 & $1.70(1.15,2.53)$ & 0.008 & 128 & $1.24(0.91,1.68)$ & 0.171 & 67 & $2.19(1.45,3.32)$ & 0.000 & 81 & $1.18(0.83,1.68)$ & 0.348 & 114 & $1.75(1.26,2.44)$ & 0.001 \\
\hline TT & 18 & 21 & $1.85(0.95,3.57)$ & 0.069 & 4 & $0.90(0.29,2.76)$ & 0.850 & 20 & $1.64(0.84,3.19)$ & 0.147 & 5 & $1.32(0.47,3.74)$ & 0.595 & 17 & $2.12(1.05,4.27)$ & 0.035 & 8 & $1.00(0.42,2.38)$ & 0.999 \\
\hline $\mathrm{TC} / \mathrm{TT}$ & 169 & 149 & $1.40(1.04,1.88)$ & 0.028 & 71 & $1.61(1.09,2.37)$ & 0.016 & 148 & $1.27(0.95,1.71)$ & 0.109 & 72 & $2.07(1.38,3.11)$ & 0.000 & 98 & $1.27(0.91,1.78)$ & 0.154 & 122 & $1.65(1.20,2.28)$ & 0.002 \\
\hline \multicolumn{20}{|c|}{$X P C$ rs 2228000} \\
\hline $\mathrm{CC}$ & 228 & 145 & Reference & & 56 & Reference & & 141 & Reference & & 60 & Reference & & 98 & Reference & & 103 & Reference & \\
\hline CT & 174 & 139 & $1.28(0.94,1.74)$ & 0.119 & 59 & $1.39(0.91,2.10)$ & 0.124 & 149 & $1.41(1.04,1.91)$ & 0.028 & 49 & $1.06(0.69,1.63)$ & 0.782 & 91 & $1.24(0.88,1.76)$ & 0.226 & 107 & $1.36(0.97,1.90)$ & 0.074 \\
\hline $\mathrm{TT}$ & 28 & 28 & $1.69(0.95,2.99)$ & 0.074 & 23 & $3.43(1.83,6.44)$ & 0.000 & 34 & $2.08(1.20,3.60)$ & 0.009 & 17 & $2.42(1.24,4.75)$ & 0.010 & 26 & $2.39(1.32,4.34)$ & 0.004 & 25 & $2.02(1.12,3.64)$ & 0.020 \\
\hline $\mathrm{CT} / \mathrm{TT}$ & 202 & 167 & $1.33(0.99,1.78)$ & 0.058 & 82 & $1.67(1.13,2.46)$ & 0.010 & 183 & $1.49(1.12,2.00)$ & 0.007 & 66 & $1.25(0.84,1.86)$ & 0.280 & 117 & $1.38(0.99,1.92)$ & 0.056 & 132 & $1.45(1.05,2.00)$ & 0.023 \\
\hline \multicolumn{20}{|c|}{$X P C$ rs 2228001} \\
\hline $\mathrm{AA}$ & 161 & 127 & Reference & & 66 & Reference & & 139 & Reference & & 54 & Reference & & 96 & Reference & & 97 & Reference & \\
\hline $\mathrm{AC}$ & 213 & 139 & $0.81(0.59,1.12)$ & 0.205 & 56 & $0.64(0.42,0.96)$ & 0.033 & 142 & $0.77(0.56,1.05)$ & 0.095 & 53 & $0.73(0.48,1.13)$ & 0.160 & 86 & $0.67(0.47,0.95)$ & 0.026 & 109 & $0.85(0.60,1.19)$ & 0.341 \\
\hline $\mathrm{CC}$ & 56 & 46 & $1.02(0.64,1.61)$ & 0.940 & 16 & $0.68(0.36,1.27)$ & 0.225 & 43 & $0.87(0.55,1.38)$ & 0.548 & 19 & $1.00(0.54,1.85)$ & 0.996 & 33 & $0.97(0.59,1.61)$ & 0.916 & 29 & $0.83(0.49,1.40)$ & 0.483 \\
\hline $\mathrm{AC} / \mathrm{CC}$ & 269 & 185 & $0.86(0.64,1.16)$ & 0.319 & 72 & $0.65(0.44,0.95)$ & 0.027 & 185 & $0.79(0.59,1.06)$ & 0.115 & 72 & $0.79(0.53,1.18)$ & 0.252 & 119 & $0.73(0.52,1.02)$ & 0.064 & 138 & $0.85(0.61,1.17)$ & 0.317 \\
\hline \multicolumn{20}{|c|}{$E R C C 2 / X P D$ rs 50872} \\
\hline $\mathrm{CC}$ & 290 & 192 & Reference & & 77 & Reference & & 195 & Reference & & 74 & Reference & & 126 & Reference & & 143 & Reference & \\
\hline $\mathrm{CT}$ & 126 & 105 & $1.24(0.90,1.70)$ & 0.186 & 55 & $1.62(1.08,2.43)$ & 0.020 & 113 & $1.31(0.96,1.80)$ & 0.089 & 47 & $1.45(0.95,2.21)$ & 0.087 & 79 & $1.42(1.00,2.03)$ & 0.049 & 81 & $1.29(0.91,1.82)$ & 0.154 \\
\hline TT & 14 & 15 & $1.68(0.79,3.57)$ & 0.180 & 6 & $1.63(0.60,4.37)$ & 0.336 & 16 & $1.77(0.84,3.72)$ & 0.134 & 5 & $1.41(0.49,4.08)$ & 0.522 & 10 & $1.69(0.73,3.91)$ & 0.223 & 11 & $1.62(0.72,3.68)$ & 0.247 \\
\hline $\mathrm{CT} / \mathrm{TT}$ & 140 & 120 & $1.28(0.94,1.74)$ & 0.113 & 61 & $1.62(1.09,2.39)$ & 0.017 & 129 & $1.36(1.01,1.84)$ & 0.046 & 52 & $1.44(0.96,2.17)$ & 0.082 & 89 & $1.45(1.03,2.04)$ & 0.032 & 92 & $1.32(0.95,1.84)$ & 0.104 \\
\hline
\end{tabular}

* Adjusted by age and menopausal status; $\mathrm{Ca}$, case; $\mathrm{Co}$, control.

genotype) was associated with estrogen receptor positive breast cancer [22]. In all, these studies suggest that patients harboring the XPC rs2228000 T allele have a higher risk of breast cancer. Furthermore, the significance of this association was confirmed by the result of the metaanalysis.

Our study revealed that $X P C$ rs2228001 was not a risk factor for breast cancer, and this was confirmed by our meta-analysis. Our subgroup analysis revealed that postmenopausal females with $\mathrm{AC}$ or $\mathrm{AC} / \mathrm{CC}$ genotype have a lower breast cancer risk. To our knowledge, this is the first study reporting these results, which should be verified by further work.

Postmenopausal females with XPA rs1800975 carrying one or two A alleles have a higher breast cancer risk than those with GG genotype, consistent with reports on populations of northern Chinese [23] and South Korean women [24]. On the other hand, a functional study showed that the XPA rs1800975 G allele increased promoter activity [25] leading to increased $X P A$ protein concentration [26]. Therefore, XPA rs1800975 AA genotype was recognized as a risk factor for lung cancer [27]. It is interesting to see contrasting results among different kinds of cancer, suggesting the susceptibility of $X P A$ rs 1800975 to cancer risk may be dependent on cancer type.

ERCC1 variant rs11615 (C19007T) is a C>T synonymous polymorphism in exon 4 (Asn118Asn), converting a high-usage codon AAC to a low-usage codon AAU. This case-control study revealed the susceptibility of carriers of ERCC1 variant rs11615 to increased risk of breast cancer, consistent with previous observations that ERCC1 rs11615 was associated with reduced mRNA [28] and protein [29] expression levels, and consequently impaired DNA repair capacity [28]. Therefore, ERCC1 rs11615 T allele carriers (CT/TT) exhibited reduced ERCC1 expression and higher breast cancer risk, which was consistent with our results. This 
Table 5: Effects of five SNPs on breast cancer risk as stratified by expression of ER, PR, and HER-2

\begin{tabular}{|c|c|c|c|c|c|c|c|c|c|c|c|c|c|c|c|c|c|c|c|}
\hline \multirow[b]{2}{*}{ Genotype } & \multirow[b]{2}{*}{ Co } & \multicolumn{3}{|c|}{ ER (-) } & \multicolumn{3}{|c|}{ ER $(+)$} & \multicolumn{3}{|c|}{ PR (-) } & \multicolumn{3}{|c|}{ PR (+) } & \multicolumn{3}{|c|}{ HER-2 (-) } & \multicolumn{3}{|c|}{ HER-2 (+) } \\
\hline & & $\mathrm{Ca}$ & OR $(95 \% \mathrm{CI})^{*}$ & $\begin{array}{c}P \\
\text { value }\end{array}$ & $\mathrm{Ca}$ & OR $(95 \% \text { CI })^{*}$ & $\begin{array}{c}P \\
\text { value }\end{array}$ & $\mathrm{Ca}$ & OR $(95 \% \mathrm{CI})^{*}$ & $\begin{array}{c}P \\
\text { value }\end{array}$ & $\mathrm{Ca}$ & OR $(95 \% \text { CI })^{*}$ & $\begin{array}{c}P \\
\text { value }\end{array}$ & $\mathrm{Ca}$ & OR $(95 \% \mathrm{CI})^{*}$ & $\begin{array}{c}P \\
\text { value }\end{array}$ & $\mathrm{Ca}$ & OR $(95 \% \text { CI })^{*}$ & $\begin{array}{c}P \\
\text { value }\end{array}$ \\
\hline \multicolumn{20}{|c|}{$X P A$ rs 1800975} \\
\hline GG & 93 & 51 & Reference & & 64 & Reference & & 61 & Reference & & 54 & Reference & & 27 & Reference & & 88 & Reference & \\
\hline GA & 231 & 86 & $0.67(0.44,1.03)$ & 0.067 & 149 & $0.93(0.64,1.36)$ & 0.718 & 109 & $0.72(0.49,1.08)$ & 0.113 & 126 & $0.93(0.62,1.39)$ & 0.720 & 46 & $0.69(0.41,1.18)$ & 0.177 & 189 & $0.86(0.60,1.22)$ & 0.399 \\
\hline $\mathrm{AA}$ & 106 & 35 & $0.60(0.36,1.01)$ & 0.055 & 65 & $0.89(0.57,1.39)$ & 0.602 & 42 & $0.61(0.37,0.99)$ & 0.044 & 58 & $0.94(0.59,1.49)$ & 0.786 & 24 & $0.78(0.42,1.45)$ & 0.432 & 76 & $0.76(0.50,1.15)$ & 0.196 \\
\hline $\mathrm{GA} / \mathrm{AA}$ & 337 & 121 & $0.65(0.44,0.98)$ & 0.038 & 214 & $0.92(0.64,1.33)$ & 0.670 & 151 & $0.69(0.47,1.00)$ & 0.053 & 184 & $0.94(0.64,1.38)$ & 0.747 & 70 & $0.73(0.44,1.20)$ & 0.212 & 265 & $0.83(0.60,1.16)$ & 0.284 \\
\hline \multicolumn{20}{|c|}{$E R C C I \mathrm{rs} 11615$} \\
\hline $\mathrm{CC}$ & 261 & 98 & Reference & & 132 & Reference & & 119 & Reference & & 111 & Reference & & 47 & Reference & & 183 & Reference & \\
\hline $\mathrm{TC}$ & 151 & 70 & $1.23(0.85,1.78)$ & 0.276 & 125 & $1.63(1.18,2.24)$ & 0.003 & 82 & $1.16(0.82,1.64)$ & 0.414 & 113 & $1.78(1.28,2.48)$ & 0.001 & 48 & $1.73(1.10,2.72)$ & 0.018 & 147 & $1.38(1.03,1.87)$ & 0.034 \\
\hline TT & 18 & 4 & $0.58(0.19,1.76)$ & 0.333 & 21 & $2.28(1.17,4.44)$ & 0.015 & 11 & $1.34(0.61,2.94)$ & 0.463 & 14 & $1.81(0.87,3.77)$ & 0.114 & 2 & $0.66(0.15,2.95)$ & 0.585 & 23 & $1.80(0.94,3.44)$ & 0.075 \\
\hline $\mathrm{TC} / \mathrm{TT}$ & 169 & 74 & $1.16(0.81,1.66)$ & 0.432 & 146 & $1.68(1.24,2.29)$ & 0.001 & 93 & $1.17(0.84,1.64)$ & 0.352 & 127 & $1.76(1.28,2.43)$ & 0.001 & 50 & $1.61(1.03,2.51)$ & 0.037 & 170 & $1.41(1.06,1.88)$ & 0.018 \\
\hline \multicolumn{20}{|c|}{$X P C$ rs 2228000} \\
\hline $\mathrm{CC}$ & 228 & 84 & Reference & & 117 & Reference & & 96 & Reference & & 105 & Reference & & 41 & Reference & & 160 & Reference & \\
\hline CT & 174 & 67 & $1.06(0.72,1.54)$ & 0.773 & 131 & $1.48(1.08,2.04)$ & 0.016 & 88 & $1.23(0.86,1.75)$ & 0.257 & 110 & $1.38(0.99,1.92)$ & 0.059 & 41 & $1.32(0.82,2.12)$ & 0.257 & 157 & $1.30(0.97,1.75)$ & 0.082 \\
\hline TT & 28 & 21 & $2.27(1.20,4.26)$ & 0.011 & 30 & $2.15(1.22,3.78)$ & 0.008 & 28 & $2.62(1.45,4.73)$ & 0.001 & 23 & $1.83(1.00,3.34)$ & 0.049 & 15 & $3.09(1.50,6.36)$ & 0.002 & 36 & $1.93(1.12,3.31)$ & 0.017 \\
\hline $\mathrm{CT} / \mathrm{TT}$ & 202 & 88 & $1.21(0.85,1.72)$ & 0.299 & 161 & $1.58(1.16,2.14)$ & 0.004 & 116 & $1.41(1.01,1.96)$ & 0.045 & 133 & $1.44(1.05,1.98)$ & 0.026 & 56 & $1.55(0.99,2.42)$ & 0.055 & 193 & $1.39(1.04,1.84)$ & 0.025 \\
\hline \multicolumn{20}{|c|}{$X P C$ rs 2228001} \\
\hline $\mathrm{AA}$ & 161 & 72 & Reference & & 121 & Reference & & 94 & Reference & & 99 & Reference & & 43 & Reference & & 150 & Reference & \\
\hline $\mathrm{AC}$ & 213 & 79 & $0.81(0.55,1.19)$ & 0.277 & 116 & $0.72(0.52,1.00)$ & 0.051 & 90 & $0.71(0.49,1.01)$ & 0.055 & 105 & $0.80(0.57,1.13)$ & 0.202 & 42 & $0.74(0.46,1.20)$ & 0.220 & 153 & $0.76(0.56,1.04)$ & 0.085 \\
\hline $\mathrm{CC}$ & 56 & 21 & $0.80(0.45,1.42)$ & 0.444 & 41 & $0.97(0.60,1.55)$ & 0.894 & 28 & $0.81(0.48,1.37)$ & 0.436 & 34 & $0.99(0.60,1.62)$ & 0.958 & 12 & $0.77(0.38,1.58)$ & 0.482 & 50 & $0.94(0.60,1.47)$ & 0.790 \\
\hline $\mathrm{AC} / \mathrm{CC}$ & 269 & 100 & $0.81(0.56,1.16)$ & 0.251 & 157 & $0.77(0.57,1.05)$ & 0.098 & 118 & $0.73(0.52,1.02)$ & 0.066 & 139 & $0.84(0.61,1.16)$ & 0.284 & 54 & $0.75(0.48,1.18)$ & 0.213 & 203 & $0.80(0.60,1.07)$ & 0.133 \\
\hline \multicolumn{20}{|c|}{$E R C C 2 / X P D$ rs 50872} \\
\hline $\mathrm{CC}$ & 290 & 89 & Reference & & 180 & Reference & & 113 & Reference & & 156 & Reference & & 58 & Reference & & 211 & Reference & \\
\hline $\mathrm{CT}$ & 126 & 73 & $1.86(1.28,2.71)$ & 0.001 & 87 & $1.10(0.79,1.53)$ & 0.588 & 85 & $1.71(1.20,2.43)$ & 0.003 & 75 & $1.09(0.77,1.55)$ & 0.621 & 36 & $1.43(0.89,2.28)$ & 0.136 & 124 & $1.33(0.98,1.81)$ & 0.069 \\
\hline TT & 14 & 10 & $2.44(1.04,5.73)$ & 0.040 & 11 & $1.27(0.56,2.86)$ & 0.565 & 14 & $2.61(1.20,5.68)$ & 0.016 & 7 & $0.93(0.37,2.36)$ & 0.882 & 3 & $1.08(0.30,3.90)$ & 0.905 & 18 & $1.80(0.88,3.72)$ & 0.110 \\
\hline $\mathrm{CT} / \mathrm{TT}$ & 140 & 83 & $1.92(1.34,2.76)$ & 0.000 & 98 & $1.11(0.81,1.53)$ & 0.518 & 99 & $1.80(1.28,2.52)$ & 0.001 & 82 & $1.08(0.77,1.51)$ & 0.658 & 39 & $1.39(0.88,2.19)$ & 0.157 & 142 & $1.38(1.03,1.85)$ & 0.033 \\
\hline
\end{tabular}

*Adjusted by age and menopausal status; Ca, case; Co, control.

association was supported by the pooled results of this meta-analysis and the study carried out on a population in China [30]. Additionally, in our study the increased risk of breast cancer linked to ERCC1 rs11615 more prominent in postmenopausal females and patients with positive expression of PR and ER, indicating the risk conveyed by this polymorphism to breast cancer in menopausal females [30].

$E R C C 2 / X P D$ rs50872 is a $\mathrm{C} / \mathrm{T}$ polymorphism in intron 4 of $X P D$. This case-control study linked ERCC2/ $X P D$ rs50872 to increased breast cancer risk and showed the polymorphism was more prevalent in the patients with tumor size T3-T4, negative lymph node involvement and patients with $\mathrm{ER}^{-}$and $\mathrm{PR}^{-}$expression, which was consistent with the conclusions in a South Korean population [24].

Some limitations of this study should be noted. First, the relatively small sample size may limit the statistical power to find differences among groups and therefore some associations may be missed, particularly in the multiple stratified analyses. Therefore, we carried out a meta-analysis to confirm the results of the case-control study. Second, several potential environmental factors, such as occupational exposure and diet, were not included in this study, which may influence breast cancer risk. Third, patients' clinical outcomes were not traced for the analysis of the predictive value of polymorphisms in the NER pathway. Finally, the polymorphisms included in this study were still limited, and these polymorphisms were selected based on previous knowledge of their potential functional roles in the occurrence of cancers. Analysis of a wider range of polymorphisms would provide more complete information about the associations of NER genes and breast cancer risk.

In conclusion, our study deduced that ERCC1 rs 11615 (CT or CT/TT), XPC rs2228000 (TT or CT/TT) and rs50872 (CT or CT/TT) were risk factors associated with increased breast cancer incidence, especially 
Table 6: Meta-analysis of the XPC rs2228000, rs2228001, XPA rs1800975 and ERCC1 rs11615 polymorphism on breast cancer risk

\begin{tabular}{|c|c|c|c|c|c|c|c|c|c|c|c|c|c|}
\hline \multirow[b]{2}{*}{ Variables } & \multirow{2}{*}{$\begin{array}{c}\text { Cases/ } \\
\text { controls }\end{array}$} & \multicolumn{3}{|c|}{ Homozygote vs. wild type } & \multicolumn{3}{|c|}{ Heterozygote vs. wild type } & \multicolumn{3}{|c|}{ Dominant model } & \multicolumn{3}{|c|}{ Recessive model $^{\mathrm{d}}$} \\
\hline & & OR(95\% CI) & $\begin{array}{c}P \\
\text { value }\end{array}$ & $P_{h}^{\mathrm{b}}$ & OR(95\% CI $)$ & $\begin{array}{c}P \\
\text { value }\end{array}$ & $\boldsymbol{P}_{h}^{\mathrm{b}}$ & OR $(95 \%$ CI $)$ & $\begin{array}{c}P \\
\text { value }\end{array}$ & $\boldsymbol{P}_{h}^{\mathrm{b}}$ & OR(95\% CI) & $\begin{array}{c}P \\
\text { value }\end{array}$ & $P_{h}^{\mathrm{b}}$ \\
\hline \multicolumn{14}{|c|}{$X P C$ rs 2228000} \\
\hline Total & $3897 / 4877^{\mathrm{a}}$ & $1.28(1.08-1.52)$ & 0.004 & 0.228 & $1.01(0.92,1.10)$ & 0.867 & 0.291 & $1.02(0.89,1.17)^{\mathrm{c}}$ & 0.766 & 0.030 & $1.25(1.06,1.47)$ & 0.008 & 0.521 \\
\hline \multicolumn{14}{|l|}{ Ethnicities } \\
\hline Caucasian & $570 / 676$ & $0.95(0.59,1.51)$ & 0.811 & 0.979 & $0.86(0.68,1.08)$ & 0.196 & 0.983 & $0.87(0.69,1.09)$ & 0.219 & 0.991 & $1.01(0.64,1.59)$ & 0.981 & 0.990 \\
\hline Asian & $1068 / 1052$ & $1.73(1.30,2.31)$ & 0.000 & 0.384 & $1.26(1.05,1.51)$ & 0.015 & 0.803 & $1.37(1.15,1.64)$ & 0.000 & 0.475 & $1.52(1.16,1.99)$ & 0.002 & 0.349 \\
\hline Other & $2259 / 3149$ & $1.13(0.89,1.43)$ & 0.318 & 0.748 & $0.97(0.86,1.08)$ & 0.520 & 0.809 & $0.97(0.88,1.08)$ & 0.597 & 0.557 & $1.14(0.90,1.43)$ & 0.287 & 0.674 \\
\hline \multicolumn{14}{|l|}{$\begin{array}{l}\text { Source of } \\
\text { control }\end{array}$} \\
\hline PB & $2364 / 3220$ & $1.37(1.11,1.68)$ & 0.003 & 0.107 & $1.09(0.97,1.22)$ & 0.148 & 0.198 & $1.15(0.94,1.42)^{\mathrm{c}}$ & 0.171 & 0.033 & $1.29(1.06,1.57)$ & 0.010 & 0.266 \\
\hline HB & $1533 / 1657$ & $1.12(0.82,1.51)$ & 0.485 & 0.642 & $0.91(0.79,1.04)$ & 0.149 & 0.931 & $0.91(0.80,1.04)$ & 0.164 & 0.717 & $1.15(0.86,1.56)$ & 0.349 & 0.656 \\
\hline \multicolumn{14}{|c|}{$X P C$ rs 2228001} \\
\hline Total & $6176 / 6955$ & $0.99(0.89,1.10)$ & 0.850 & 0.343 & $0.97(0.90,1.05)$ & 0.430 & 0.206 & $0.97(0.91,1.05)$ & 0.470 & 0.180 & $1.01(0.91,1.11)$ & 0.869 & 0.444 \\
\hline \multicolumn{14}{|l|}{ Ethnicities } \\
\hline Caucasian & $1714 / 1613$ & $0.85(0.70,1.05)$ & 0.369 & 0.369 & $0.91(0.78,1.05)$ & 0.194 & 0.608 & $0.89(0.78,1.03)$ & 0.112 & 0.462 & $0.90(0.75,1.09)$ & 0.279 & 0.485 \\
\hline African & $814 / 753$ & $0.90(0.60,1.35)$ & 0.649 & 0.649 & $0.94(0.77,1.16)$ & 0.567 & 0.308 & $0.94(0.77,1.14)$ & 0.512 & 0.420 & $0.93(0.63,1.37)$ & 0.716 & 0.513 \\
\hline Asian & $1068 / 1052$ & $1.14(0.87,1.49)$ & 0.196 & 0.196 & $1.01(0.59,1.75)^{\mathrm{c}}$ & 0.962 & 0.004 & $1.04(0.63,1.71)^{\mathrm{c}}$ & 0.894 & 0.005 & $1.10(0.86,1.42)$ & 0.446 & 0.826 \\
\hline Other & $2580 / 3537$ & $1.04(0.89,1.22)$ & 0.298 & 0.298 & $0.99(0.88,1.10)$ & 0.810 & 0.792 & $1.00(0.90,1.11)$ & 0.998 & 0.891 & $1.06(0.92,1.21)$ & 0.453 & 0.130 \\
\hline \multicolumn{14}{|l|}{$\begin{array}{l}\text { Source of } \\
\text { control }\end{array}$} \\
\hline PB & $4587 / 5222$ & $0.95(0.84,1.08)$ & 0.441 & 0.377 & $0.95(0.87,1.04)$ & 0.258 & 0.081 & $0.95(0.88,1.03)^{\mathrm{b}}$ & 0.230 & 0.056 & $0.98(0.87,1.10)$ & 0.691 & 0.795 \\
\hline $\mathrm{HB}$ & $1589 / 1733$ & $1.10(0.89,1.35)$ & 0.371 & 0.336 & $1.03(0.89,1.20)$ & 0.700 & 0.738 & $1.05(0.91,1.21)$ & 0.523 & 0.971 & $1.09(0.91,1.32)$ & 0.346 & 0.122 \\
\hline \multicolumn{14}{|c|}{$X P A$ rs 1800975} \\
\hline Total & $2619 / 2663$ & $0.92(0.65,1.31)$ & 0.649 & 0.003 & $1.07(0.78,1.48)$ & 0.663 & 0.001 & $1.03(0.74,1.42)$ & 0.873 & 0.000 & $0.94(0.84,1.06)$ & 0.303 & 0.190 \\
\hline \multicolumn{14}{|l|}{ Ethnicities } \\
\hline Asian & $1407 / 1409$ & $0.82(0.54,1.26)$ & 0.372 & 0.018 & $0.95(0.60,1.51)$ & 0.841 & 0.002 & $0.91(0.58,1.43)$ & 0.686 & 0.001 & $0.83(0.70,0.99)$ & 0.039 & 0.539 \\
\hline Other & $1212 / 1254$ & $1.22(0.94,1.59)$ & 0.129 & 0.255 & $1.30(1.01,1.66)$ & 0.039 & 0.821 & $1.27(1.01,1.60)$ & 0.046 & 0.795 & $1.05(0.89,1.23)$ & 0.585 & 0.23 \\
\hline
\end{tabular}

ERCC1 rs 11615

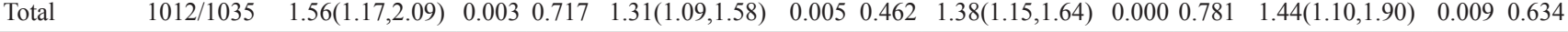

${ }^{a}$ rs2228000 cases/controls are not include the studies of Smith(b) and Perez-Mayoral

${ }^{\mathrm{b}} P_{\mathrm{h}}$ value of Q-test for heterogeneity test.

'Random-effects model was used when a $P$ value $<0.05$ for heterogeneity test; otherwise, fixed-effects model was used.

${ }^{\mathrm{d}}$ Available data by Tatemichi et al was used in stratified analyses by cancer type, ethnicity and source of control for the recessive model comparison.

PB: population based control studies; HB: hospital based control studies.

in postmenopausal women. The risk conferred by polymorphisms in NER pathway genes for breast cancer among females with different menopausal status should be evaluated in a larger cohort study.

\section{MATERIALS AND METHODS}

\section{Study subjects}

For the case-control association study, from January 2008 to January 2015 in Nanjing First Hospital, Nanjing Medical University, China, we enrolled 450 female patients histologically diagnosed with breast cancer, and 430 age-matched healthy females, who visited the same hospital for routine physical examination, were enrolled as non-cancer controls. All participants were from the same geographic region. The clinical characteristics of each subject, including smoking, drinking, and other cancer history, were collected via a questionnaire and written informed consents were obtained from all participants. Participants were enrolled in this study with no limitation for the smoking and drinking or not, and finally, there were less than ten individuals with a history of smoking and drinking, which may be attributed to the lifestyle 
of Chinese females. We excluded these samples as unrepresentative of the population before genotyping. The protocol of this study was approved by the Institutional Review Board of Nanjing First Hospital.

\section{Genotyping of polymorphisms}

Genotyping was performed as we described previously $[31,32]$. The genotyping for all the polymorphisms was performed by Sequenom MassARRAY RS1000 according to the standard protocol. Multiplexed SNP MassEXTENDED assay was designed by Sequenom MassARRAY Assay Design 3.0 Software [33]. Finally, data management and analysis were performed by Sequenom Typer 4.0 Software [33, 34].

\section{Immunohistochemistry (IHC) assay}

The expression of ER, PR, and HER-2 in paraffinembedded tumor tissue samples was evaluated by immunohistochemistry (IHC) assay, as we described previously $[31,32]$.

\section{Meta-analysis of polymorphisms in $X P A$ (rs1800975), XPC (rs2228000, rs2228001), and ERCC1 (rs11615)}

Meta-analysis was performed to confirm the polymorphisms identified as breast cancer risk factors by our case-control study. Four polymorphisms (XPA (rs1800975), XPC (rs2228000, rs2228001), and ERCC1 (rs11615)) were evaluated for breast cancer risk using pooled data from this study and available published studies. The ERCC2/XPD rs50873 was ruled out for lack of available published data.

To identify relevant studies, we searched PubMed and Embase databases using the keywords 'XPA,' 'XPC' or 'ERCC1', 'polymorphism,' and 'breast cancer' (updated to March 31, 2016). The papers were limited to studies on human subjects and published in English. In addition, references listed in any reviews were manually searched to ensure all relevant studies were included. Then, we evaluated the collected publications by screening the titles and abstracts. All studies which matched the following inclusion criteria were retrieved: (i) evaluated at least one of these four polymorphisms (XPC rs2228000, rs2228001, $X P A$ rs1800975, and ERCC1 rs11615) and risk of breast cancer; (ii) from a case-control association study; and (iii) with available genotype frequencies.

All data complying with the selection criteria were extracted by two authors (B. H., and T. X.), independently. For each study, the following characteristics were extracted: the first author's last name, country of origin, patient ethnicity, the number of genotyped cases and controls, and the result of this case-control study was also applied for the meta-analysis. For the stratified analysis, subgroup analysis was performed according to ethnicity, which were categorized as Caucasian, Asian, and other; those with mixed ethnicities were categorized as others. In addition, subgroup analysis based on the origin of controls was also applied according to the participants of enrolled studies from population or hospital.

\section{Statistical analysis}

For the case-control association study, the statistical analysis of genotype distribution was performed by $\chi^{2}$ test. The risk of polymorphisms was evaluated by odds ratios (OR) and 95\% confidence intervals (CIs), which were calculated using a logistic regression model. $P$ value $<0.05$ was considered to have statistically significant difference. Software SPSS 11.0 for Windows (SPSS, Chicago, IL, USA) was used for the statistics.

For the meta-analysis, the overall risk associated with a polymorphism to breast cancer was measured by ORs with 95\% CIs based on different genetic models [Rare allele homozygote (RR), heterozygous (WR), and $\mathrm{RR}+\mathrm{WR}$ vs. wild-type homozygote (WW) genotypes]. Stratified analyses were performed by ethnicity. The $Z$ test was performed to calculate the pooled OR, and a $P$ value $<0.05$ was considered as significant. The $\chi^{2}$ based Q statistical test was used to evaluated the heterogeneity across the enrolled studies [36], and a $P$ value of heterogeneity $\left(P_{\mathrm{h}}\right)<0.05$ was considered significant. The random-effects model was used when there was marked heterogeneity across all the studies; otherwise, the fixedeffects model was used [37]. All statistical tests for this meta-analysis were performed with STATA version 10.0 (Stata Corporation College Station, TX, USA).

\section{ACKNOWLEDGMENTS}

This study was supported by grants from Nanjing Medical Science and Technology Development Foundation to B.H (no. JQX13003, QRX11254, and QYK11175) and Y. P (no. QRX11255). We are grateful to Prof. Hong-Guang Xie, General Clinical Research Center, Nanjing First Hospital, Nanjing Medical University, Jiangsu, China, for his critical review, scientific editing, and constructive comments.

\section{CONFLICTS OF INTEREST}

The authors have no conflicts of interests to declare.

\section{REFERENCES}

1. Torre LA, Bray F, Siegel RL, Ferlay J, Lortet-Tieulent J, Jemal A. Global cancer statistics, 2012. CA Cancer J Clin. 2015; 65:87-108. 
2. Davis JD, Lin SY. DNA damage and breast cancer. World journal of clinical oncology. 2011; 2:329-338.

3. Wood RD, Mitchell M, Lindahl T. Human DNA repair genes, 2005. Mutation research. 2005; 577:275-283.

4. Lambert WC, Lambert MW. Development of effective skin cancer treatment and prevention in xeroderma pigmentosum. Photochem Photobiol. 2015; 91:475-483.

5. Zhao F, Shang Y, Zeng C, Gao D, Li K. Association of single nucleotide polymorphisms of DNA repair genes in NER pathway and susceptibility to pancreatic cancer. Int J Clin Exp Pathol. 2015; 8:11579-11586.

6. Mittal RD, Mandal RK. Genetic variation in nucleotide excision repair pathway genes influence prostate and bladder cancer susceptibility in North Indian population. Indian J Hum Genet. 2012; 18:47-55.

7. Wheless L, Kistner-Griffin E, Jorgensen TJ, Ruczinski I, Berthier-Schaad Y, Kessing B, Hoffman-Bolton J, Francis L, Shugart YY, Strickland PT, Kao WH, Alani RM, Smith MW, et al. A community-based study of nucleotide excision repair polymorphisms in relation to the risk of non-melanoma skin cancer. J Invest Dermatol. 2012; 132:1354-1362.

8. Scarbrough PM, Weber RP, Iversen ES, Brhane Y, Amos CI, Kraft P, Hung RJ, Sellers TA, Witte JS, Pharoah P, Henderson BE, Gruber SB, Hunter DJ, et al. A CrossCancer Genetic Association Analysis of the DNA Repair and DNA Damage Signaling Pathways for Lung, Ovary, Prostate, Breast, and Colorectal Cancer. Cancer epidemiology, biomarkers \& prevention : a publication of the American Association for Cancer Research, cosponsored by the American Society of Preventive Oncology. 2016; 25:193-200.

9. Ramos JM, Ruiz A, Colen R, Lopez ID, Grossman L, Matta JL. DNA repair and breast carcinoma susceptibility in women. Cancer. 2004; 100:1352-1357.

10. Latimer JJ, Johnson JM, Kelly CM, Miles TD, BeaudryRodgers KA, Lalanne NA, Vogel VG, Kanbour-Shakir A, Kelley JL, Johnson RR, Grant SG. Nucleotide excision repair deficiency is intrinsic in sporadic stage I breast cancer. Proc Natl Acad Sci U S A. 2010; 107:21725-21730.

11. Yan Y, Liang H, Light M, Li T, Deng Y, Li M, Li S, Qin X. XPD Asp312Asn and Lys751Gln polymorphisms and breast cancer susceptibility: a meta-analysis. Tumour biology. 2014; 35:1907-1915

12. Xu XM, Xie LC, Yuan LL, Hu XL, Jin JQ, Niu YM. Association of xeroderma pigmentosum complementation group G Asp1104His polymorphism with breast cancer risk: A cumulative meta-analysis. Molecular and clinical oncology. 2014; 2:1177-1181.

13. Pabalan N, Francisco-Pabalan O, Sung L, Jarjanazi H, Ozcelik H. Meta-analysis of two ERCC2 (XPD) polymorphisms, Asp312Asn and Lys751Gln, in breast cancer. Breast cancer research and treatment. 2010; 124:531-541.
14. Jiang Z, Li C, Xu Y, Cai S, Wang X. Associations between XPD polymorphisms and risk of breast cancer: a metaanalysis. Breast cancer research and treatment. 2010; 123:203-212.

15. Na N, Dun E, Ren L, Li G. Association between ERCC5 gene polymorphisms and breast cancer risk. International journal of clinical and experimental pathology. 2015; 8:3192-3197.

16. Zhu G, Wang L, Guo H, Lu L, Yang S, Wang T, Guo H, Wang H, Min J, Yang K, Chen X, Liu Y, Wang Z, Su H. DNA repair genes XRCC1 and ERCC1 polymorphisms and the risk of sporadic breast cancer in Han women in the Gansu Province of China. Genet Test Mol Biomarkers. 2015; 19:387-393.

17. Lu J, Zhao H, Li S, Tian Z, Zhu X, Wang H, Fu H. Correlation of rs1799793 polymorphism in ERCC2 and the clinical response to platinum-based chemotherapy in patients with triple negative breast cancer. International journal of clinical and experimental medicine. 2015; 8:2934-2938.

18. Wang T, Wang H, Guo H, Yang S, Zhu G, Guo H, Wang L, Li Y, Yang K, Li H, Min J, Li X, Hu Q, Wang Y, Liu $\mathrm{Y}$, Zhang B, et al. Polymorphisms in the DNA repair gene ERCC2/XPD and breast cancer risk: a HapMap-based case-control study among Han Women in a Chinese lessdeveloped area. Genetic testing and molecular biomarkers. 2014; 18:703-710.

19. Wang H, Wang T, Guo H, Zhu G, Yang S, Hu Q, Du Y, Bai X, Chen X, Su H. Association analysis of ERCC5 gene polymorphisms with risk of breast cancer in Han women of northwest China. Breast cancer. 2016; 23:479-485.

20. Sugasawa K, Ng JM, Masutani C, Iwai S, van der Spek PJ, Eker AP, Hanaoka F, Bootsma D, Hoeijmakers JH. Xeroderma pigmentosum group $\mathrm{C}$ protein complex is the initiator of global genome nucleotide excision repair. Mol Cell. 1998; 2:223-232.

21. He J, Shi TY, Zhu ML, Wang MY, Li QX, Wei QY. Associations of Lys939Gln and Ala499Val polymorphisms of the XPC gene with cancer susceptibility: a meta-analysis. Int J Cancer. 2013; 133:1765-1775.

22. Yang $X$, Liu $D, W u ~ H$, Kang $H$, Pang $H$, Huang D, Sha X, Wang E, Wang Z, Wei M. Association of XPC polymorphisms with susceptibility and clinical outcome to chemotherapy in breast cancer patients. Cancer science. 2012; 103:1207-1214.

23. Ding P, Yang Y, Cheng L, Zhang X, Cheng L, Li C, Cai J. The relationship between seven common polymorphisms from five DNA repair genes and the risk for breast cancer in northern Chinese women. PloS one. 2014; 9:e92083.

24. Han W, Kim KY, Yang SJ, Noh DY, Kang D, Kwack K. SNP-SNP interactions between DNA repair genes were associated with breast cancer risk in a Korean population. Cancer. 2012; 118:594-602. 
25. Wang F, He Y, Guo H, Li J, Yang Y, Wu Z, Zheng H, Wu T. Genetic variants of nucleotide excision repair genes are associated with DNA damage in coke oven workers. Cancer epidemiology, biomarkers \& prevention : a publication of the American Association for Cancer Research, cosponsored by the American Society of Preventive Oncology. 2010; 19:211-218.

26. Park JY, Park SH, Choi JE, Lee SY, Jeon HS, Cha SI, Kim CH, Park JH, Kam S, Park RW, Kim IS, Jung TH. Polymorphisms of the DNA repair gene xeroderma pigmentosum group A and risk of primary lung cancer. Cancer epidemiology, biomarkers \& prevention : a publication of the American Association for Cancer Research, cosponsored by the American Society of Preventive Oncology. 2002; 11:993-997.

27. Lou Y, Li R, Zhang Y, Zhong R, Pei J, Xiong L, Zhang $\mathrm{X}$, Han B. XPA gene rs1800975 single nucleotide polymorphism and lung cancer risk: a meta-analysis. Tumour Biol. 2014; 35:6607-6617.

28. Park DJ, Stoehlmacher J, Zhang W, Tsao-Wei DD, Groshen S, Lenz HJ. A Xeroderma pigmentosum group D gene polymorphism predicts clinical outcome to platinum-based chemotherapy in patients with advanced colorectal cancer. Cancer Res. 2001; 61:8654-8658.

29. Yu JJ, Mu C, Lee KB, Okamoto A, Reed EL, BostickBruton F, Mitchell KC, Reed E. A nucleotide polymorphism in ERCC1 in human ovarian cancer cell lines and tumor tissues. Mutat Res. 1997; 382:13-20.

30. Yang Z, Fang X, Pei X, Li H. Polymorphisms in the ERCC1 and XPF genes and risk of breast cancer in a Chinese population. Genet Test Mol Biomarkers. 2013; 17:700-706.
31. He BS, Pan YQ, Lin K, Ying HQ, Wang F, Deng QW, Sun HL, Gao TY, Wang SK. Evaluation the susceptibility of five polymorphisms in microRNA-binding sites to female breast cancer risk in Chinese population. Gene. 2015; 573:160-165.

32. He B, Pan Y, Xu Y, Deng Q, Sun H, Gao T, Wang S. Associations of polymorphisms in microRNAs with female breast cancer risk in Chinese population. Tumour Biol. 2015; 36:4575-4582.

33. Gabriel S, Ziaugra L, Tabbaa D. SNP genotyping using the Sequenom MassARRAY iPLEX platform. Curr Protoc Hum Genet. 2009; Chapter 2:Unit 212.

34. Thomas RK, Baker AC, Debiasi RM, Winckler W, Laframboise T, Lin WM, Wang M, Feng W, Zander T, MacConaill L, Lee JC, Nicoletti R, Hatton C, et al. Highthroughput oncogene mutation profiling in human cancer. Nat Genet. 2007; 39:347-351.

35. Gul AE, Keser SH, Barisik NO, Kandemir NO, Cakir C, Sensu S, Karadayi N. The relationship of cerb B 2 expression with estrogen receptor and progesterone receptor and prognostic parameters in endometrial carcinomas. Diagn Pathol. 2010; 5:13.

36. Handoll HH. Systematic reviews on rehabilitation interventions. Arch Phys Med Rehabil. 2006; 87:875.

37. Midgette AS, Wong JB, Beshansky JR, Porath A, Fleming C, Pauker SG. Cost-effectiveness of streptokinase for acute myocardial infarction: A combined meta-analysis and decision analysis of the effects of infarct location and of likelihood of infarction. Med Decis Making. 1994; 14:108-117. 